\title{
Precision Timing Measurements for High Energy Photons
}

\author{
Dustin Anderson $^{\mathrm{a}, *}$, Artur Apreysan ${ }^{\mathrm{a}}$, Adi Bornheim ${ }^{\mathrm{a}}$, Javier Duarte ${ }^{\mathrm{a}}$, Harvey Newman $^{\mathrm{a}}$, Cristian Pena ${ }^{\mathrm{a}}$, Anatoly Ronzhin ${ }^{\mathrm{b}}$, Maria \\ Spiropulu $^{\mathrm{a}}$, Jason Trevor ${ }^{\mathrm{a}}, \mathrm{Si} \mathrm{Xie}^{\mathrm{a}}$, Ren-Yuan $\mathrm{Zhu}^{\mathrm{a}}$ \\ ${ }^{a}$ California Institute of Technology, 1200 E California Blvd, Pasadena, CA 91125, USA \\ ${ }^{b}$ Fermi National Accelerator Laboratory, P.O. Box 500, Batavia, IL 60510, USA
}

\begin{abstract}
Particle colliders operating at high luminosities present challenging environments for high energy physics event reconstruction and analysis. We discuss how timing information, with a precision on the order of $10 \mathrm{ps,} \mathrm{can} \mathrm{aid} \mathrm{in} \mathrm{the} \mathrm{reconstruction} \mathrm{of} \mathrm{physics} \mathrm{events}$ under such conditions. We present calorimeter based timing measurements from test beam experiments in which we explore the ultimate timing precision achievable for high energy photons or electrons of $10 \mathrm{GeV}$ and above. Using a prototype calorimeter consisting of a $1.7 \times 1.7 \times 1.7 \mathrm{~cm}^{3}$ LYSO crystal cube, read out by micro-channel plate PMTs (MCP-PMTs), we demonstrate a time resolution of $(33.5 \pm 2.1)$ ps for an incoming beam energy of $32 \mathrm{GeV}$. In a second measurement, using a $2.5 \times 2.5 \times 20 \mathrm{~cm}^{3}$ LYSO crystal placed perpendicularly to the electron beam, we achieve a time resolution of (59 \pm 11$)$ ps using a beam energy of 4 $\mathrm{GeV}$. We also present timing measurements made using a shashlik-style calorimeter cell made of LYSO and tungsten plates, and demonstrate that the apparatus achieves a time resolution of (54 \pm 5$)$ ps for an incoming beam energy of $32 \mathrm{GeV}$.
\end{abstract}

Keywords: precision timing, calorimetry, high energy physics, picosecond

\section{Introduction}

Current and future high energy particle colliders are capable of providing instantaneous luminosities of $10^{34} \mathrm{~cm}^{-2} \mathrm{~s}^{-1}$ and ${ }_{31}$ above [1]. The high center of mass energy, the large number of ${ }_{32}$ simultaneous collisions of beam particles in the experiments, ${ }_{33}$ and the very high repetition rates of the collision events pose ${ }_{34}$ huge challenges for physics event reconstruction. They result in ${ }_{35}$ extremely high particle fluxes, causing high occupancies in the ${ }_{36}$ particle detectors operating at these machines. To reconstruct the physics events, the detectors must make as much information as possible available on the final state particles.

Precise timing of particles from high energy beam collisions is one promising method for successfully reconstructing ${ }_{41}$ physics events. Measuring particle time of flight (TOF) with a precision on the order of 10 ps would allow one to associate in- ${ }_{43}$ dividual particles to primary collision vertices with a precision of about $1 \mathrm{~cm}$ or less, allowing spurious particles to be rejected from the physics events of interest.

In high energy hadron collisions, about one third of the typical particle flux is detected as photons stemming from neutral meson decays, identified by means of their interaction with scintillating material in the detector. Due to the abundance of photons in hadron collisions, it is viable to consider calorimeter based TOF detectors, in which the energies and TOF of incoming high energy photons are measured simultaneously via scintillators interfaced with fast photodetectors. Calorimeter based options for time measurement could provide an alternative to

\footnotetext{
* Corresponding Author

Email address: djanders@caltech.edu (Dustin Anderson)
}

dedicated TOF devices on particle detectors, simplifying detector design.

One candidate scintillator for a calorimeter based TOF detector is cerium-doped lutetium-yttrium oxyortho-silicate (LYSO). LYSO crystals are desirable for timing applications because of their fast rise time $(<72 \mathrm{ps}[2])$ and high scintillation light yield (in excess of 30000 photons/MeV [3]). Their radiation hardness also makes them ideal for use in high energy physics experiments.

The time resolution attained by a crystal calorimeter is influenced by a number of independent factors [4] (see Fig. 1). In addition to contributions from the DAQ and the photodetector(s), the resolution is affected by jitter in the scintillation process and in the optical transit time of scintillation photons in the crystal. For high energy photons incident on the calorimeter, variation in the conversion depth also impacts the total time resolution.

Figure 1: Schematic showing various contributions to the resolution obtained when measuring time using crystal scintillation light. [4]

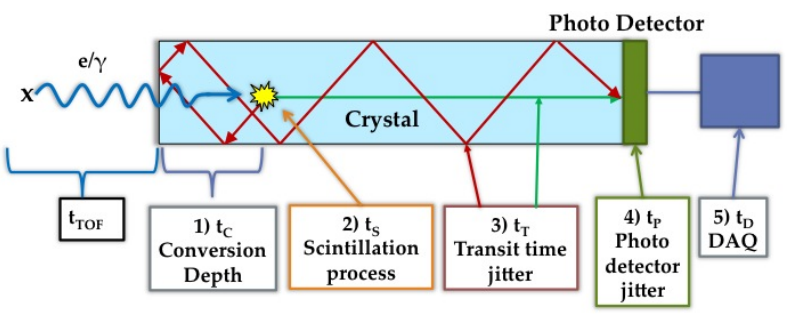

To test the viability of a calorimeter based TOF detector, one should isolate the effects of these different factors and deter- 
mine the contribution of each one to the time resolution. In this study we aim to investigate the effects of the scintillation process and the optical transit time on the TOF resolution. To best isolate these effects, we use beams of high energy electrons (which do not need to convert before beginning the scintillation process) instead of photons, and make use of the fastest photodetectors and readout electronics available.

\section{Experimental Setup}

\subsection{TOF resolution using a $1.7 \times 1.7 \times 1.7 \mathrm{~cm}^{3}$ LYSO crystal}

Measurements of electron TOF resolution using LYSO crystals were carried out at the Fermilab Test Beam Facility (FTBF) using beams of electrons with energies varying between $4 \mathrm{GeV}$ and $32 \mathrm{GeV}$. The experimental setup is shown in Fig. 2 and schematically in Fig. 3. The electron beam impinges upon a small LYSO cube $\left(1.7 \times 1.7 \times 1.7 \mathrm{~cm}^{3}\right)$ that is coupled optically to a Photek 240 micro-channel plate PMT (MCP-PMT). Lead bricks are placed in front of the MCP-PMT to shield it from direct hits by the electron beam. A second Photek 240 device is placed upstream to provide a time reference. A Cherenkov detector, further upstream, discriminates between electrons and other, unwanted particles in the incoming beam. The three MCP-PMTs and the Cherenkov detector are connected to $\mathrm{a}_{79}$ DRS4 digitizer unit [5] for readout. A small piece of scintillator, coupled to two XP-2020 PMTs and placed in the beam- ${ }_{81}$ line, is used as an external trigger for the DRS4 unit. Coincidence between the two XP-2020 PMTs is required in order for ${ }_{83}$ an event to be recorded.

Figure 2: Photo of the setup used to measure electron time of flight with a 85 $1.7 \times 1.7 \times 1.7 \mathrm{~cm}^{3}$ LYSO crystal interfaced with an MCP-PMT photodetector 86 and read out using a DRS4 digitizer unit. The beam arrives from the top edge ${ }_{87}$ of the picture. A Hamamatsu R3809U MCP-PMT is placed downstream of the setup in order to tag beam particles not fully absorbed by the LYSO crystal, but ${ }^{88}$ it is not used to reject events.

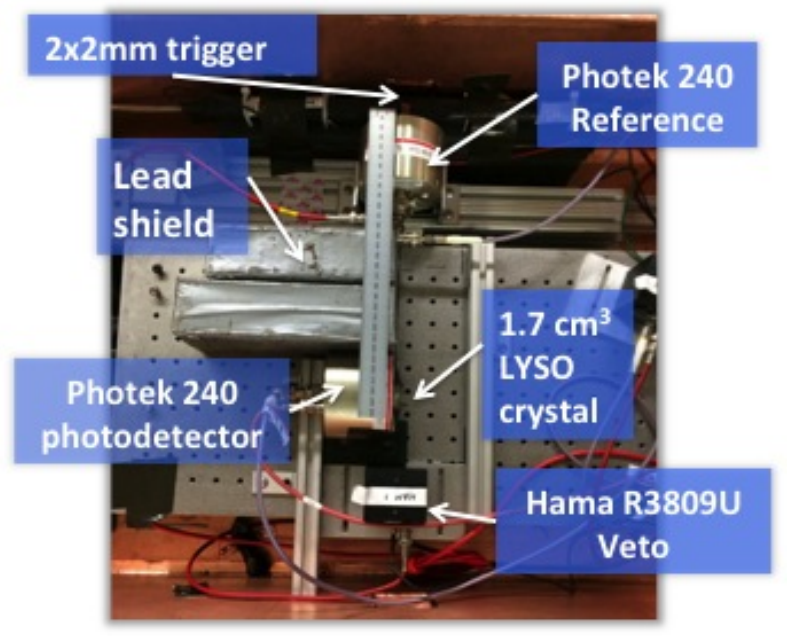

The DRS4 unit has a sampling rate of $5 \mathrm{GHz}$. To determine 106
Figure 3: Diagram of the setup used to measure electron time of flight with a $1.7 \times 1.7 \times 1.7 \mathrm{~cm}^{3}$ LYSO crystal interfaced with an MCP-PMT photodetector and read out using a DRS4 digitizer unit.

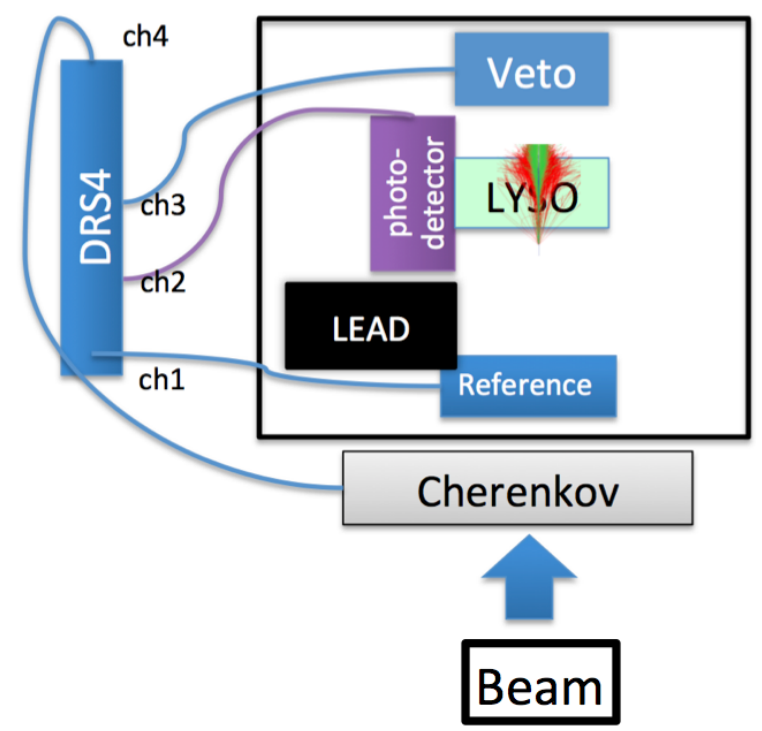

measured for many incident laser pulses, and the RMS of the distribution of time differences is taken as a measure of the intrinsic DRS4 time resolution. This method yields a time resolution for the DRS4 unit of about $5 \mathrm{ps}$. The time resolution of the reference time detector and DRS4 combined is approximately $20 \mathrm{ps}[6]$.

In events that pass the DRS4 external trigger and are not rejected by the Cherenkov detector, the TOF between the reference MCP-PMT and the MCP-PMT attached to the LYSO crystal is measured. The time is extracted from the reference MCPPMT using a gaussian fit to the digitized pulse shape (see Fig. 4). The time is extracted from the MCP-PMT attached to the LYSO crystal using a constant-fraction fit to the rising edge of the scintillation pulse (see Fig. 5). The difference between these two numbers is taken as the TOF. The TOF distribution for all selected events is fit with a gaussian, whose RMS is taken as the TOF resolution.

\subsection{TOF resolution using a $2.5 \times 2.5 \times 20 \mathrm{~cm}^{3}$ LYSO crystal}

In a second measurement, the TOF resolution is measured using a large $2.5 \times 2.5 \times 20 \mathrm{~cm}^{3}$ LYSO crystal placed perpendicularly to the incoming electron beam. A picture of this setup is shown in Fig. 6. Photek 240 MCP-PMTs are optically coupled to the ends of the crystal, and a Hamamatsu R3809U MCP-PMT is placed downstream as a time reference. The three MCP-PMTs are read out using the DRS4 unit, and the time stamps are extracted from the pulse shapes using the gaussian fit for the Hamamatsu MCP-PMT and the constant-fraction fit for the Photek MCP-PMTs. To alleviate smearing of the TOF due to uncertainty in the transverse position of the electron beam, the times extracted from the two Photek MCP-PMTs are averaged to obtain a single value for the arrival time of the scintillation light. 
Figure 4: Digitized pulse from the reference MCP-PMT, coarse-grained to show the pulse structure. A gaussian fit to the pulse is used to extract a reference time stamp for the TOF measurement.

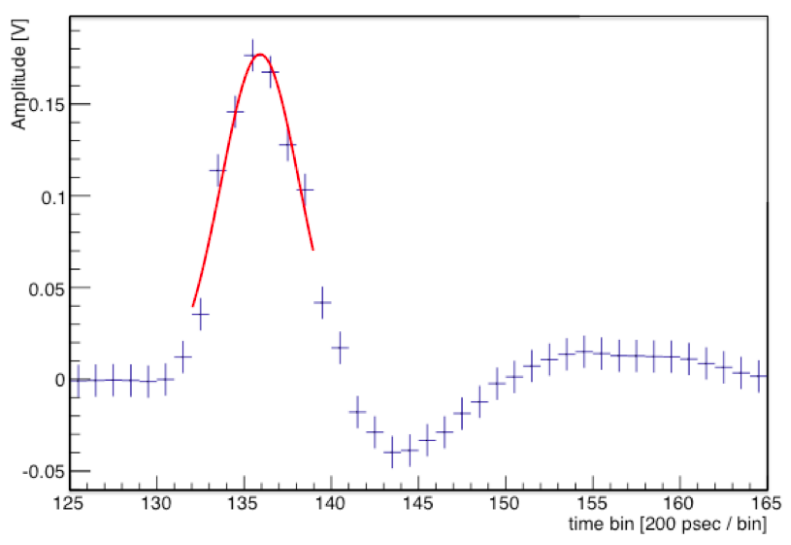

Figure 5: Digitized pulse from the MCP-PMT receiving scintillation light from the $1.7 \times 1.7 \times 1.7 \mathrm{~cm}^{3}$ LYSO crystal, coarse-grained to show the pulse structure. A constant-fraction fit to the pulse is used to extract a time stamp for the TOF measurement.

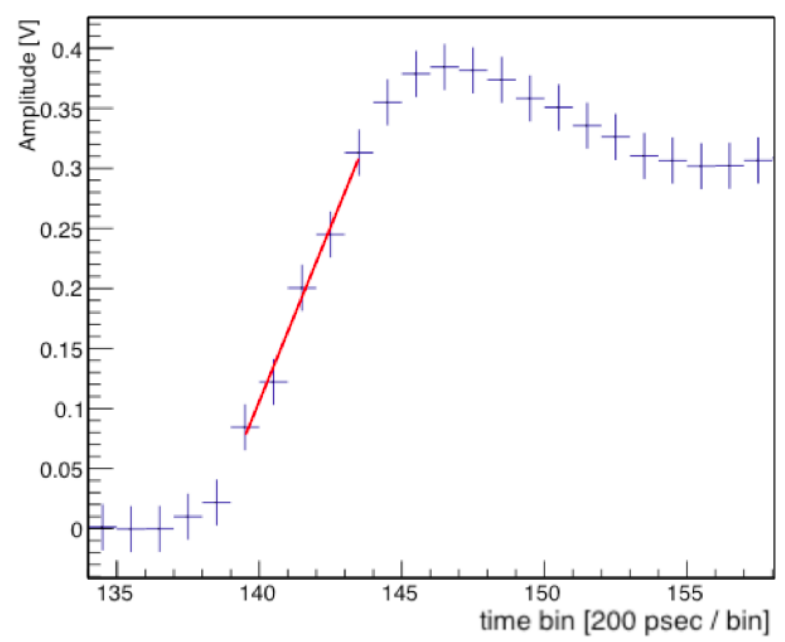

126

\subsection{TOF resolution using a $1.4 \times 1.4 \times 13 \mathrm{~cm}^{3}$ LYSO/Tungsten $^{127}$ shashlik cell}

In a third measurement, the TOF resolution is measured us- 132 ing a $1.2 \times 1.2 \times 16 \mathrm{~cm}^{3}$ shashlik style [1] calorimeter cell, con-133 structed using alternating plates of tungsten and LYSO crys-134 tal (see Fig. 7). This apparatus is placed along the direction ${ }_{135}$ of the electron beam. For the TOF measurement, Hamamatsu ${ }_{136}$ R3809U MCP-PMTs are placed on either side of the shashlik ${ }_{137}$ setup, in direct contact with a single LYSO tile in the stack. ${ }_{138}$ A Photek 240 MCP-PMT is placed downstream of the shash-139 lik setup as a time reference. The time is extracted from the ${ }_{140}$ Photek MCP-PMT using the gaussian fit, and the time from ${ }_{141}$ each Hamamatsu MCP-PMT is extracted using the constant-142 fraction fit. The time values extracted from the two sides of the $\mathrm{e}_{143}$ LYSO tile are averaged for the calculation of the TOF.
Figure 6: Photo of the setup used to measure electron time of flight with a $2.5 \times 2.5 \times 20 \mathrm{~cm}^{3}$ LYSO crystal interfaced with an MCP-PMT photodetector and read out using a DRS4 digitizer unit. The beam arrives from the top edge of the picture.

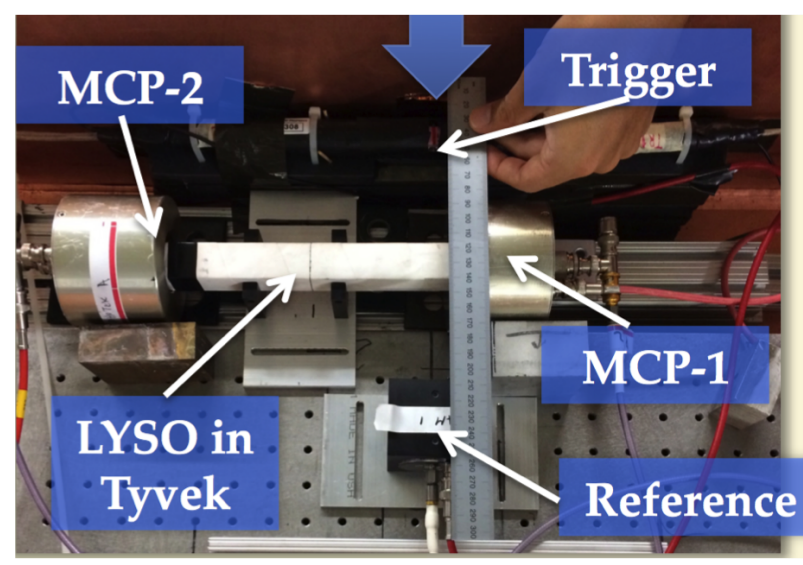

Figure 7: Diagram of the shashlik style calorimeter cell used to measure electron TOF. The cell consists of alternating plates of LYSO crystal and tungsten, separated by protective sheets of TYVEK paper. Y11 wavelength shifting fibers run through the center of the cell and collect light from the LYSO plates for measurement of the incoming particle energy, but they are not used in the TOF measurement.

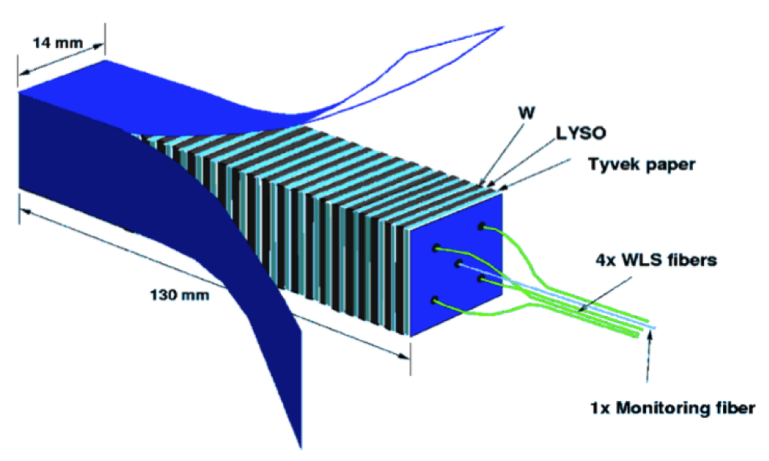

\section{Results}

On examination of the scintillation pulses obtained using the setup with the $1.7 \times 1.7 \times 1.7 \mathrm{~cm}^{3}$ LYSO crystal, we observe in many of the pulses an initial 'spike' feature that occurs slightly before the main body of the pulse (see Fig. 11). The cause of this spike is hypothesized to be direct hits of beam particles on the entry window of the MCP-PMT coupled to the LYSO crystal. Because this feature occurs in a large fraction of selected events in this study, we conclude that direct hits on the MCPPMT window have an impact on the measured time resolution, tending to decrease it. In a later test beam experiment, the TOF measurement was repeated using Hamamatsu R3809U MCPPMTs, which have a smaller active area than the Photek 240 and are thus less prone to direct hits. Additional lead shielding is also put in place to further shield the MCP-PMT on the LYSO crystal.

The TOF distribution obtained using the modified setup is shown in Fig. 8 for a beam energy of $32 \mathrm{GeV}$. The TOF resolution obtained is $(33.5 \pm 2.1)$ ps. 
Figure 8: TOF distribution obtained using an electron beam incident on a $1.7 \times 1.7 \times 1.7 \mathrm{~cm}^{3}$ LYSO crystal interfaced with a Hamamatsu MCP-PMT. The distribution is obtained using a $32 \mathrm{GeV}$ electron beam. The TOF resolution is taken to be the width of a gaussian fit to the TOF distribution and is determined here to be $(33.5 \pm 2.1) \mathrm{ps}$.

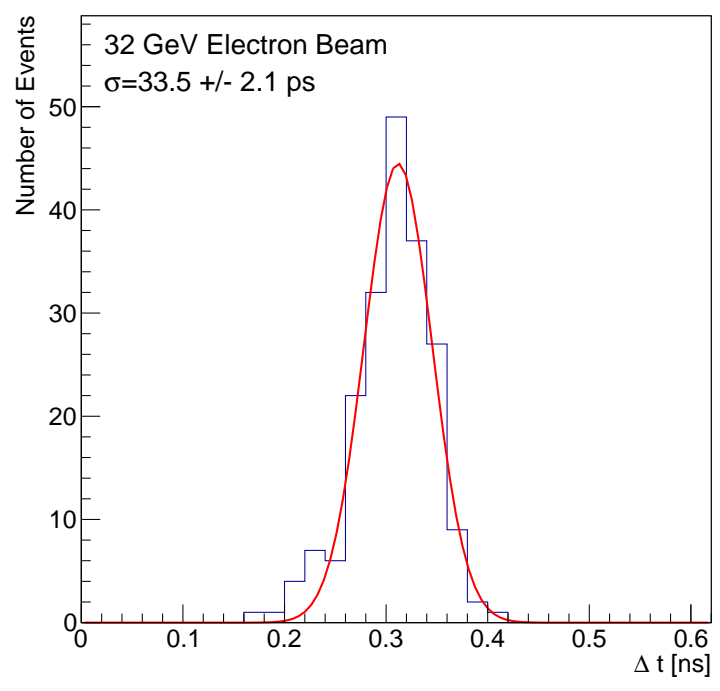

The TOF distribution obtained using the $2.5 \times 2.5 \times 20 \mathrm{~cm}^{3}$
LYSO crystal is shown in Fig. 9 for a $4 \mathrm{GeV}$ electron beam. The TOF resolution obtained from this distribution is $(59 \pm 11)$ ps.

The TOF distribution obtained using the LYSO/tungsten shashlik cell is shown in Fig. 10 for a $32 \mathrm{GeV}$ electron beam. The TOF resolution obtained from this distribution is $(54 \pm 5)$ ps.

\section{Discussion}

The measurements in Sections 2.1, 2.2 and 2.3 demonstrate that the goal of sub- $100 \mathrm{ps}$ time resolution can be achieved using LYSO crystals coupled to photodetectors. This is an encouraging result that points to the potential usefulness of LYSO crystals in calorimeter based TOF detectors. Further study is necessary in order to verify that the time resolution scales in the expected way with the energy of the incident particles, and to arrive at an estimate for the time resolution achievable at very high energies.

[1] A. Bornheim, "Evolution of the CMS ECAL response, R\&D studies on new scintillators and possible design options for electromagnetic calorimetry at the HL-LHC', Proceedings of the CHEF 2013, Paris, France, Eds. J.-C. Brient, R. Salerno, and Y. Sirois.

[2] S. Seifert et al., "Accurate measurement of the rise and decay times of fast scintillators with solid state photon counters," JINST 7 P09004 (2012).

[3] C. Wanarak et al., "Light yield non-proportionality and energy resolution of $\mathrm{Lu}_{1.8} \mathrm{Y}_{0.2} \mathrm{SiO}_{5}: \mathrm{Ce}$ and $\mathrm{LaCl}_{3}$ :Ce scintillation crystals," Advanced Materials Research Vols. 284-286 (2011) 2002-2007.

[4] A. Bornheim, "Calorimeters for precision timing measurements in high IOP Conference Series.

[5] Paul Scherrer Institute, DRS Chip Homepage, http://www.psi.ch/drs/, 12 Sept. 2014.

[6] A. Ronzhin et. al., NIM A, Vol 749 p 65-73. energy physics", CALOR 2014, Giessen, Germany, to be published in
Figure 9: TOF distribution obtained using a $4 \mathrm{GeV}$ electron beam incident on a $2.5 \times 2.5 \times 20 \mathrm{~cm}^{3}$ LYSO crystal. The TOF resolution is determined to be $(59 \pm 11) \mathrm{ps}$.

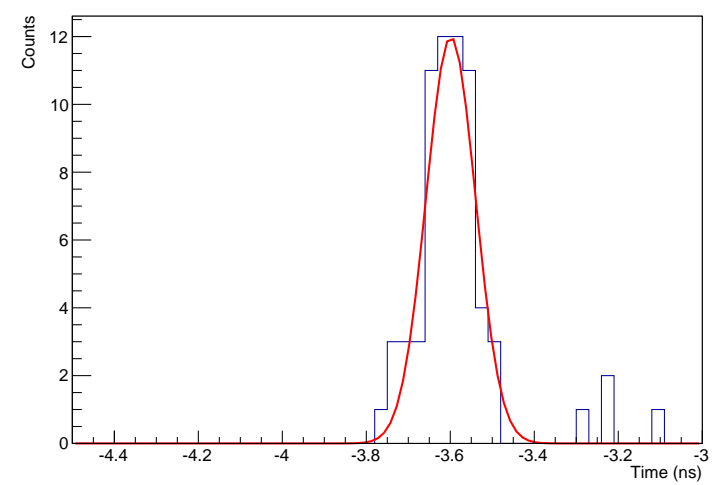

Figure 10: TOF distribution obtained using side readout of a single LYSO tile in a $1.4 \times 1.4 \times 13 \mathrm{~cm}^{3}$ LYSO/Tungsten shashlik cell on which a $32 \mathrm{GeV}$ electron beam is incident. The TOF resolution is determined to be $(54 \pm 5) \mathrm{ps}$.

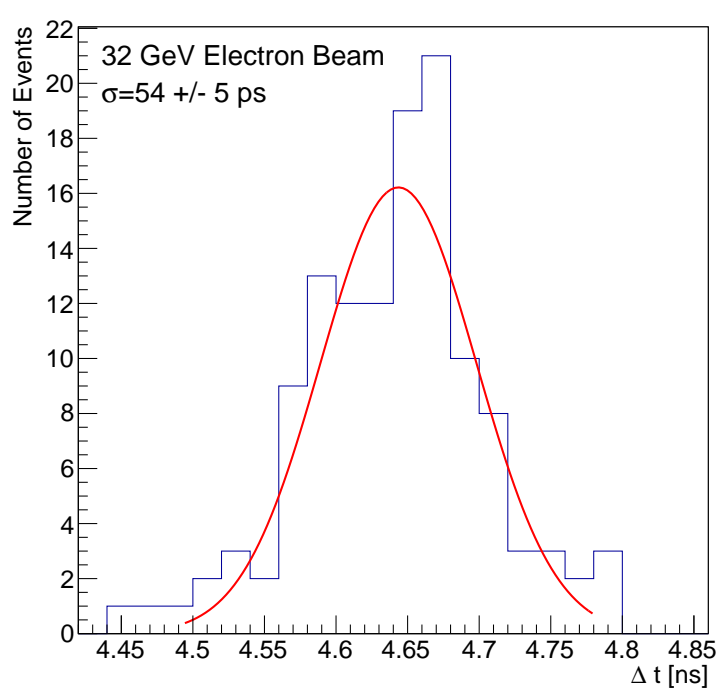

Figure 11: Example pulse from the $1.7 \times 1.7 \times 1.7 \mathrm{~cm}^{3}$ LYSO cube setup with Photek 240 MCP-PMTs. The sharp feature at the leading edge of the pulse is thought to be caused by a direct hit of a beam particle on the MCP-PMT window. To mitigate the effects of direct hits, the measurement is repeated using an MCP-PMT with a smaller active area.

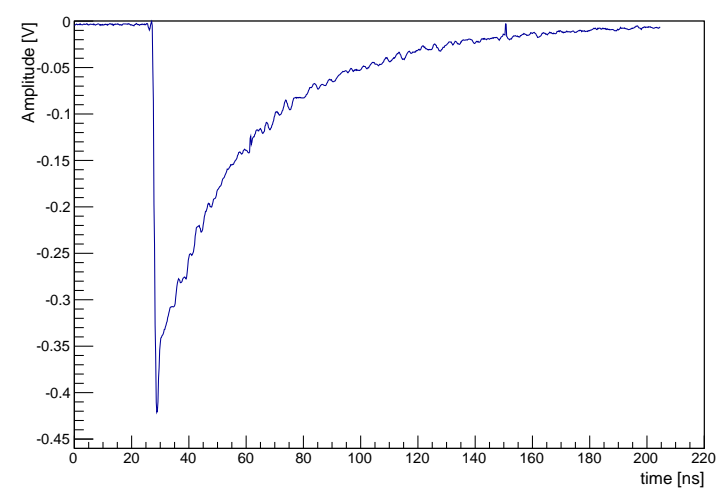

